\title{
Magnetohydrodynamic Casson Fluid Flow over an Infinite Vertical Plate with Chemical Reaction and Heat Generation
}

\author{
E. Omokhuale, M. L. Jabaka \\ Department of Mathematical Sciences, Federal University Gusau, Zamfara State, Nigeria \\ Email: emmanuelomokhuale@yahoo.com,muhammadjabaka@gmail.com
}

How to cite this paper: Omokhuale, E. and Jabaka, M.L. (2019) Magnetohydrodynamic Casson Fluid Flow over an Infinite Vertical Plate with Chemical Reaction and Heat Generation. American Journal of Computational Mathematics, 9, 187-200. https://doi.org/10.4236/ajcm.2019.93014

Received: August 19, 2019

Accepted: September 24, 2019

Published: September 27, 2019

Copyright $\odot 2019$ by author(s) and Scientific Research Publishing Inc. This work is licensed under the Creative Commons Attribution International License (CC BY 4.0).

http://creativecommons.org/licenses/by/4.0/

\begin{abstract}
In this paper, the fluid examined was electrically conducting. The presence of a uniform transverse magnetic field at the plate was also taken into cognizance. The flow was governed by a modeled coupled nonlinear system of partial differential equations (PDEs) in dimensional form which was transformed into non-dimensional form using some non-dimensional variables. Explicit finite difference method (EFDM) was employed to approximate the fluid velocity, temperature and concentration. The effects of embedded thermo physical parameters of engineering interests on the flow quantities viz. velocity, temperature, concentration field presented through graphs were also examined through a series of numerical experiments and discussed. During the course of the numerical computations, it was found that heat generation has a tendency to enhance the fluid velocity as an opposite result is seen with chemical reaction parameter. A comparison was conducted of present results with the previous literature to show the accuracy of the results.
\end{abstract}

\section{Keywords}

Heat Generation, Casson Fluid, Chemical Reaction, MHD and EFDM

\section{Introduction}

Investigations on Magnetohydrodynamic (MHD) flow of non-Newtonian fluid in a porous medium have gained the focus of many researchers. Clearly, this is because such a phenomenon is greatly found in the optimization and solidification processes of metals and their alloys. Wide applications of MHD flows of non-Newtonian fluids in a porous medium are seen in irrigation problems, heat storage beds, biological systems, textile industries, and paper and polymer com- 
posite industries. The few to mention who have contributed in this area are Kateria and Petal [1] that studied the influence of radiation and chemical reaction on MHD Casson fluid past an oscillating vertical plate embedded in a porous medium. Others are: Makinde and Mhone [2]; Pushpalatha et al. [3]; Raju et al. [4]; Kumaran et al. [5]; Reddy et al. [6].

Naturally, some non-Newtonian fluids exist like elastic solid. Casson fluid is an example of such fluids with unique characteristics. Few examples of Casson fluid are jelly, tomato sauce, honey, liquid soup and concentrated fruit juices. Human blood is also a Casson fluid due to the blood cells' chain structure and contents present in aqueous base plasma like rouleaux, fibrinogen, globulin and protein. Casson model was introduced by Casson in 1959 for the prediction of the flow behavior of pigment-oil suspensions (Casson, 1959). Some studies containing the Casson fluid phenomenon are found in the works of (Hayat et al. [7]; Ajayi et al. [8]; Raju et al. [9]; Rajakumar et al. [10], Omokhuale and Altine [11]).

The growing need for chemical reactions in chemical and hydrometallurgical industries necessitates the study of heat and mass transfer in the presence of chemical reaction. The existence of a foreign mass in a fluid causes some kind of chemical reaction represented either by itself or as mixtures with a fluid. In several chemical engineering practices, a chemical reaction exists between a foreign mass and the fluid in which the plate is moving. These processes have industrial applications such as polymer production, manufacturing of ceramic or grass ware and food processing. A chemical reaction can be categorized as either a homogeneous or heterogeneous process that depends on whether it occurs on an interface or a single phase volume reaction Raju et al. [12]. Moreover, chemical reaction effects on heat and mass transfer laminar boundary flow have been examined by many scholars e.g. (Uwanta and Omokhuale [13] Arthur et al. [14]; Biswas et al. [15]; Biswas et al. [16]).

Explicit Finite difference method (EFDM) is very useful in solving coupled non-linear partial differential equations whose solutions cannot be obtained analytically. The unsteady natural convection flow past a semi-infinite vertical plate was first solved by Hellum and Churchill [17] using an explicit finite difference method. Few researchers who have employed the EFDM are (Sharma et al. [18]; Reddy, Janardhan et al. [19]; Ahmed and Alam [20]). Recently, Mondal et al. [21] conducted a numerical exploration on inscribed MHD unsteady heat and mass transfer of Casson nanofluid flow where variable thermal conductivity, radiation and heat absorption were counterfeited using EFDM. The effect of heat generation on boundary layer fluid flow is very important because of its usefulness in engineering such as radioactive materials, fire and combustion, metal waste, reactor safety analysis, spent nuclear fuel, etc. Some scholars who have researched on heat generation with respect to several phenomena are (Jasmine et al. [22]; Animasaun et al. [23], Mohan et al. [24]).

In all the above studies mentioned, the solutions of Casson fluid were mostly 
obtained in closed form using Laplace technique. There are few situations in which the numerical schemes were employed. To the best of the author's knowledge, no investigation has been reported yet to study the effects of heat generation and chemical reaction on MHD Casson fluid flow past an infinite vertical plate numerically using EFDM. The present work aims to fill the gap in the existing literatures.

\section{Mathematical Formulation}

We consider Casson fluid over an infinite vertical plate embedded in a saturated porous medium. The flow being restricted to $y>0$, where $y$ is the coordinate which is to be measured in the normal direction to the plate. The fluid is assumed to be electrically conducting with a uniform magnetic field $B$ of strength $B_{0}$, applied in a direction perpendicular to the plate. The plate temperature is raised to $T_{w}$ which is thereafter be maintained constant.

The rheological equation of state for the Cauchy stress tensor of Casson fluid is given by,

$$
\begin{aligned}
& \tau=\tau_{0}+\mu \tau^{*} \\
& \text { where } \tau_{0}=\left\{\begin{array}{l}
2\left(\mu_{B}+\frac{p_{y}}{\sqrt{2 \omega}}\right) e_{i j}, \text { when } \omega>\omega_{c} \\
2\left(\mu_{B}+\frac{p_{y}}{\sqrt{2 \pi_{c}}}\right) e_{i j}, \text { when } \omega<\omega_{c}
\end{array}\right.
\end{aligned}
$$

where $\omega=e_{i j} e_{i j}$ and $e_{i j}$ is the $(i, j)^{\text {th }}$ component of the deformation rate.

The following assumptions were made before deriving the governing equations; incompressible flow, free convection and non-Newtonian flow, infinite vertical plate, fluid suction and magnetic field are imposed at the plate surface, the temperature and concentration of the fluid are raised to $T_{w}^{\prime}$ and $C_{w}^{\prime}$ respectively and are higher than the ambient temperature and that of fluid. In addition, the effects of variable heat generation and chemical reaction is taken into account. It is also assumed that viscous dissipation is not neglected.

The governing equations of the flow under the usual Boussinesq and boundary-layer approximation can be written as:

$$
\begin{gathered}
\frac{\partial v^{\prime}}{\partial y^{\prime}}=0 \\
\rho\left(\frac{\partial u^{\prime}}{\partial t^{\prime}}+v^{\prime} \frac{\partial u^{\prime}}{\partial y^{\prime}}\right) \\
=\mu_{B}\left(1+\frac{1}{\gamma}\right) \frac{\partial^{2} u^{\prime}}{\partial y^{\prime 2}}-\sigma B_{0}^{2} u^{\prime}-\frac{\mu \eta}{K_{1}} u^{\prime}+g \rho\left[\beta_{T}\left(T^{\prime}-T_{\infty}^{\prime}\right)+\beta_{m}\left(C^{\prime}-C_{\infty}^{\prime}\right)\right] \\
\rho C_{p}\left(\frac{\partial T^{\prime}}{\partial t^{\prime}}+v^{\prime} \frac{\partial T^{\prime}}{\partial y^{\prime}}\right)=k_{0} \frac{\partial}{\partial y^{\prime}}\left\{\left[1+m\left(T^{\prime}-T_{\infty}^{\prime}\right)\right] \frac{\partial T^{\prime}}{\partial y^{\prime}}\right\}+Q\left(T^{\prime}-T_{\infty}^{\prime}\right)+\frac{\mu}{C_{p}}\left(\frac{\partial u^{\prime}}{\partial y^{\prime}}\right)^{2} \\
\frac{\partial C^{\prime}}{\partial t^{\prime}}+v^{\prime} \frac{\partial C^{\prime}}{\partial y^{\prime}}=D \frac{\partial^{2} C^{\prime}}{\partial y^{\prime 2}}-R^{*}\left(C^{\prime}-C_{\infty}^{\prime}\right)
\end{gathered}
$$


with the following initial and boundary conditions:

$$
\begin{aligned}
& t^{\prime} \leq 0, u^{\prime}=0, T^{\prime} \rightarrow T_{\infty}^{\prime}, C^{\prime} \rightarrow C_{\infty}^{\prime} \text { for all } y^{\prime} \\
& t^{\prime}>0, u^{\prime}=u_{p}, T^{\prime}=T_{w}^{\prime}, C^{\prime}=C_{w}^{\prime} \text { at } y^{\prime}=0 \\
& u^{\prime} \rightarrow 0, T^{\prime} \rightarrow T_{\infty}^{\prime}, C^{\prime} \rightarrow C_{\infty}^{\prime} \text { as } y^{\prime} \rightarrow \infty
\end{aligned}
$$

where $u$ and $v$ are velocity components in $x^{\prime}$ and $y^{\prime}$ directions respectively, $T$ is the temperature, $t$ is the time, $\mathrm{g}$ is the acceleration due to gravity, $\beta$ is the thermal expansion coefficient, $\beta^{*}$ is the concentration expansion coefficient, $v$ is the kinematic viscosity, $D$ is the chemical molecular diffusivity, $C_{p}$ is heat capacity at constant pressure, $B_{0}$ is a constant magnetic field intensity, $\sigma$ is the electrical conductivity of the fluid, $k_{0}$ is the variable thermal conductivity, $\rho$ is the density, $\lambda_{1}$ is the Jeffery fluid, $T_{w}$ is the wall temperature, $T_{\infty}$ is the free stream temperature, $C_{w}$ is the species concentration at the plate surface, $C_{\infty}$ is the free stream concentration, $Q$ is the heat generation coefficient.

$v_{0}>0$ is the suction parameter and $v_{0}<0$ is the injection parameter. On introducing the following non-dimensionless quantities

$$
\left.y=\frac{u_{0}}{v} y^{\prime}, u_{p}=\frac{u_{p}^{\prime}}{u_{0}}, t=\frac{u_{0}^{2}}{v} t^{\prime}, u=\frac{u^{\prime}}{u_{0}}, \theta=\frac{T^{\prime}-T_{\infty}^{\prime}}{T_{w}-T_{\infty}}, C=\frac{C^{\prime}-C_{\infty}^{\prime}}{C_{w}-C_{\infty}}, \tau^{*}=\frac{\tau}{\rho u^{2}}\right\}
$$

Using (1) and (6), equations (2) - (5) are transformed to the following:

$$
\begin{gathered}
\frac{\partial u}{\partial t}-\xi \frac{\partial u}{\partial y}=\left(1+\frac{1}{\gamma}\right) \frac{\partial^{2} u}{\partial y^{2}}-M^{2} u-\frac{1}{K} u+G r \theta+G c C \\
\frac{\partial \theta}{\partial t}-\xi \frac{\partial \theta}{\partial y}=\frac{1}{P r}(1+\delta \theta) \frac{\partial^{2} \theta}{\partial y^{2}}+\frac{\delta}{\operatorname{Pr}} \frac{\partial \theta}{\partial y}+S \theta+E c\left(\frac{\partial u}{\partial y}\right)^{2} \\
\frac{\partial C}{\partial t}-\xi \frac{\partial C}{\partial y}=\frac{1}{S c} \frac{\partial^{2} C}{\partial y^{2}}-\lambda C
\end{gathered}
$$

The corresponding boundary conditions are:

$$
\left.\begin{array}{l}
u=0, \theta=0, C=0 \text { for all } y \leq 0 \\
u=u_{p}, \theta=1, C=1 \text { at } y=0 \\
u=0, \rightarrow \theta=0, C=0 \text { as } y \rightarrow \infty
\end{array}\right\}
$$

where $\gamma$ is the Casson parameter, Gr is the thermal Grashof number, $G c$ is the mass Grashof number, $S c$ is the Schmidt number, $P r$ is the Prandtl number, $M$ is the magnetic parameter, $\lambda$ is the chemical reaction parameter, $K$ is the permeability parameter, $\xi$ is the suction parameter, $S$ is the heat generation parameter, $E_{c}$ is the Eckert number. $\delta$ is a constant.

\section{Calculation Technique}

To get the difference equations, the region of the flow is divided into a grid or mesh of line parallel to $y$ axis, where $y$-axis is normal to the plate. It is considered that the plate is of height $y_{\max }=150$ i.e. $y$ varies from 1 to 150 . There are $m=150$ and $n=300$ grid spacing in the $y$-directions. It is assumed that $\Delta y$ is a constant mesh size along $y$ direction and taken as $\Delta y=0.50(0 \leq y \leq 150)$. 
With the smaller time-step, $\Delta t=0.0005$ and $u^{\prime}, T^{\prime}$ and $C^{\prime}$ represents the values of $u, \theta$ and $C$ at the end of a time step respectively.

Therefore, the nonlinear coupled partial differential Equations (7) to (9) subject to boundary conditions (10) are solved numerically using an explicit finite difference method as below:

$$
\begin{gathered}
\left(\frac{u_{i, j}^{\prime}-u_{i, j}}{\Delta t}-\xi \frac{u_{i, j+1}-u_{i, j}}{\Delta y}\right) \\
=\left(1+\frac{1}{\gamma}\right)\left[\frac{u_{i, j+1}-2 u_{i, j}+u_{i, j-1}}{(\Delta y)^{2}}\right]-M\left(u_{i, j}\right)-\frac{1}{K}\left(u_{i, j}\right)+G r\left(\theta_{i, j}\right)+G c\left(C_{i, j}\right) \\
\left(\frac{\theta_{i, j+1}^{\prime}-\theta_{i, j}}{\Delta t}-\xi \frac{\theta_{i, j+1}-\theta_{i, j}}{\Delta y}\right) \\
=\frac{1}{2 P r} \cdot\left[1+\frac{\delta}{2}\left(\theta_{i, j+1}+\theta_{i, j}\right)\right]\left[\frac{\theta_{i, j+1}-2 \theta_{i, j}+\theta_{i, j-1}}{(\Delta y)^{2}}\right] \\
+\frac{\delta}{\operatorname{Pr}}\left(\frac{\theta_{i, j+1}-\theta_{i, j}}{\Delta y}\right)^{2}+S\left(\theta_{i, j+1}+\theta_{i, j}\right)+E c\left(\frac{u_{i, j+1}-u_{i, j}}{\Delta y}\right)^{2} \\
S c\left(\frac{C_{i, j}^{\prime}-C_{i, j}}{\Delta t}-\xi \frac{C_{i, j+1}-C_{i, j}}{\Delta y}\right)=\left[\frac{C_{i, j+1}-2 C_{i, j}+C_{i, j-1}}{(\Delta y)^{2}}\right]-\frac{\lambda S c}{2}\left(C_{i, j}\right)
\end{gathered}
$$

The initial and boundary conditions become

$$
\begin{aligned}
& u_{i, 0}=0, \theta_{i, 0}=0, C_{i, 0}=0 \text { for all } i \text { except } i=0 \\
& u_{i, 0}=u_{p}, \theta_{i, 0}=1, C_{i, 0}=1 \\
& u_{L, 0}=0, \theta_{L, 0}=1, C_{L, 0}=1
\end{aligned}
$$

where $L$ corresponds to $\infty$. The suffix $i$ corresponds to $y$ and $j$ equals to $t$. consequently, $\Delta t=t_{j+1}-t_{j}$ and $\Delta y=y_{i+1}-y_{i}$.

The finite difference approximations of these equations were solved by using the values for $G r=G c=M=\delta=1, \gamma=0.5, \operatorname{Pr}=0.71, S c=0.6, S=0.2$, $E c=0.2, \lambda=0.5, K=0.5$, except where they are varied. A step size of $\Delta y=0.01$ is used for the interval $y_{\min }=0$ to $y_{\max }=5$ for a desired accuracy and a convergence criterion of $10^{-6}$ is satisfied for various parameters.

\section{Results and Discussion}

An explicit finite difference technique has been adopted to obtain the numerical results of the present model has been carried out for the different values of parameters embedded on the velocity, temperature and concentration within the boundary conditions. The finite difference approximations of these equations were solved by using the values for $G r=G c=M=\delta=1, \gamma=0.5, \operatorname{Pr}=0.71$, $S c=0.6, S=0.2, \quad E c=0.2, \lambda=0.5, K=0.5$, except where they are varied. The interval $y_{\min }=0$ to $y_{\max }=5$ for a desired accuracy and a convergence for various parameters. 


\subsection{Velocity Profiles}

Figures 1-6 show the velocity profiles with varying parameters respectively.

Figure 1 depicts the effect of Prandtl number on the velocity. It is observed that the velocity decreases with increasing Prandtl number. Influence of Hartmann number $\mathrm{M}$ on the velocity is illustrated in Figure 2, it is found that the velocity decreases with the increase in magnetic parameter. This is due to the fact that application of transverse magnetic field to an electrically conducting fluid which results in a Lorentz force (a resistive type force) similar to the drag force which leads to the deceleration of the flow. Casson parameter effect on the

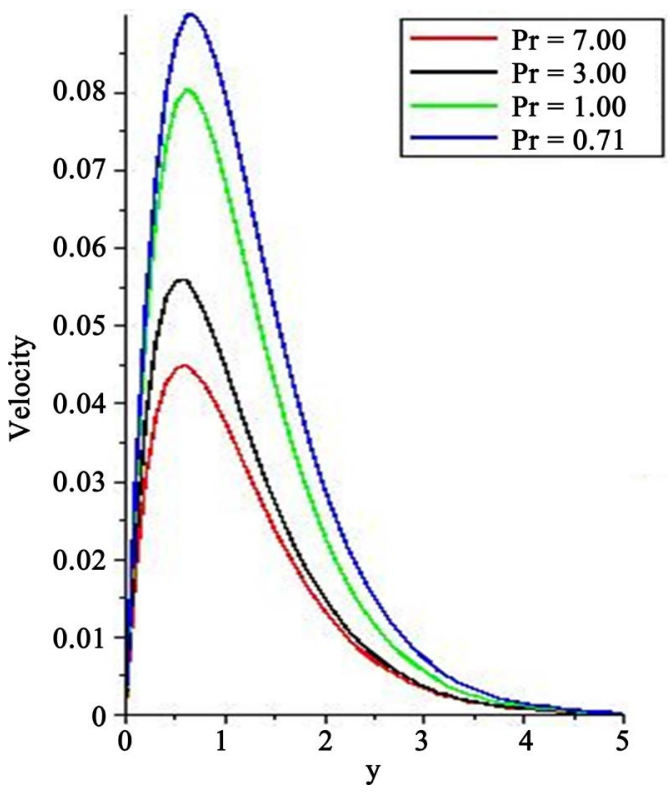

Figure 1. Velocity profiles for different values of Pr.

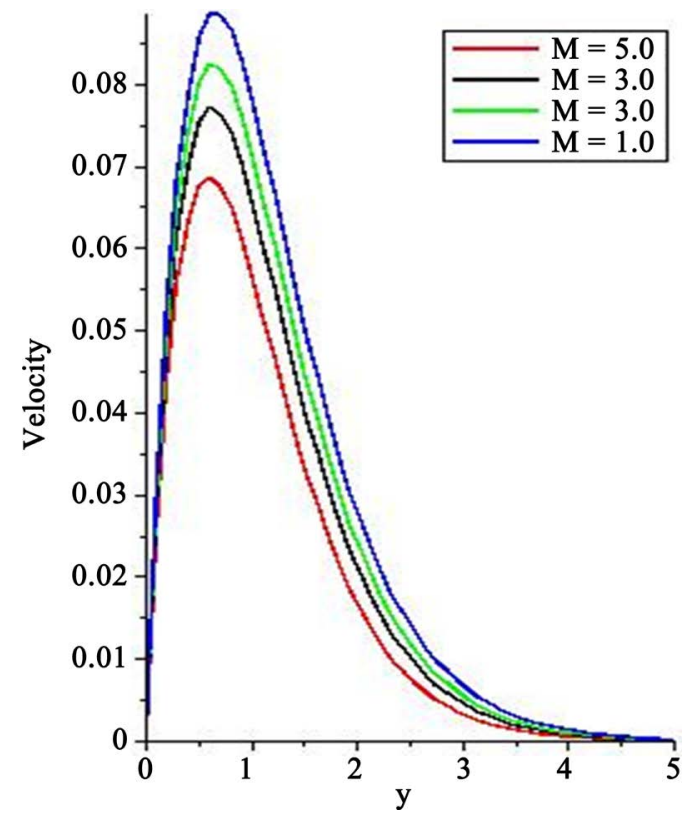

Figure 2. Velocity profiles for different values of $M$. 
velocity profile is shown in Figure 3. It is observed that, the velocity decreases with increasing Casson parameter. It is interesting to note that Casson parameter makes the velocity boundary layer thickness shorter. This occurs because of the plasticity of Casson fluid. Influence of suction parameter on the velocity is demonstrated in Figure 4. It is seen that, the velocity is lower due to an increase in suction parameter. Figure 5 represents different values of thermal Grashof number on the velocity, it is noted that, the velocity rises with increasing thermal Grashof number. In Figure 6, the effect of mass Grashof number on the velocity is presented. It is observed that, the velocity increases with increase in mass

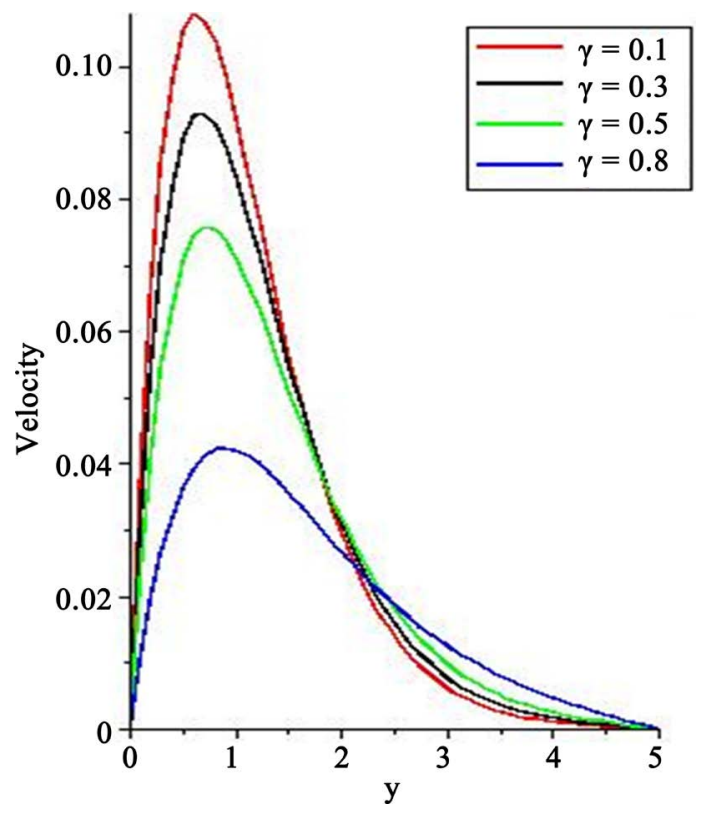

Figure 3. Velocity profiles for different values of $\gamma$.

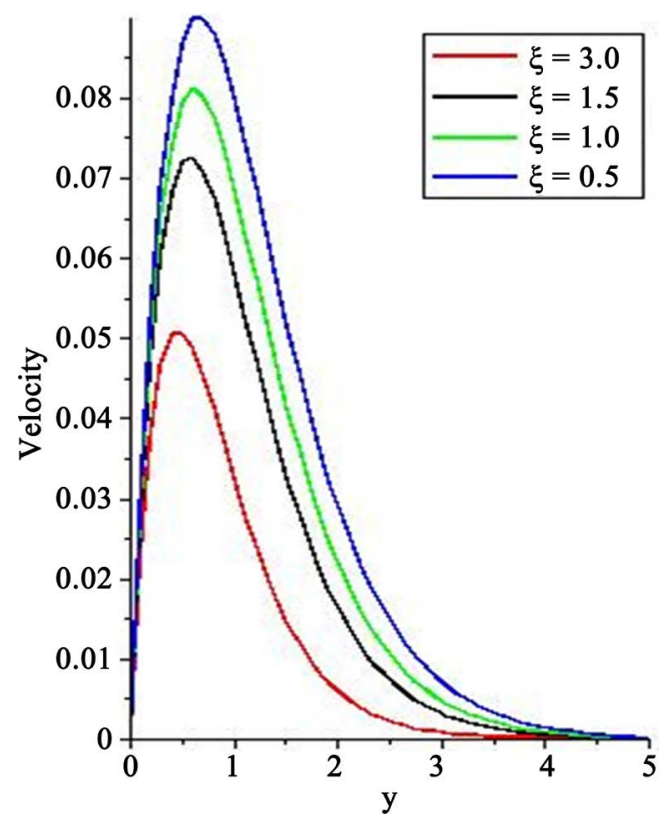

Figure 4. Velocity profiles for different values of $\xi$. 


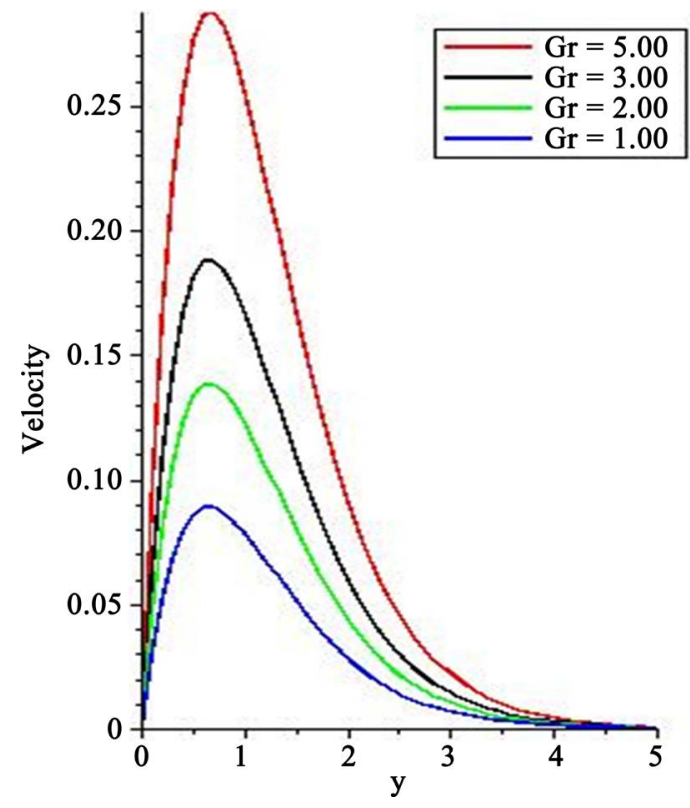

Figure 5. Velocity profiles for different values of $G r$.

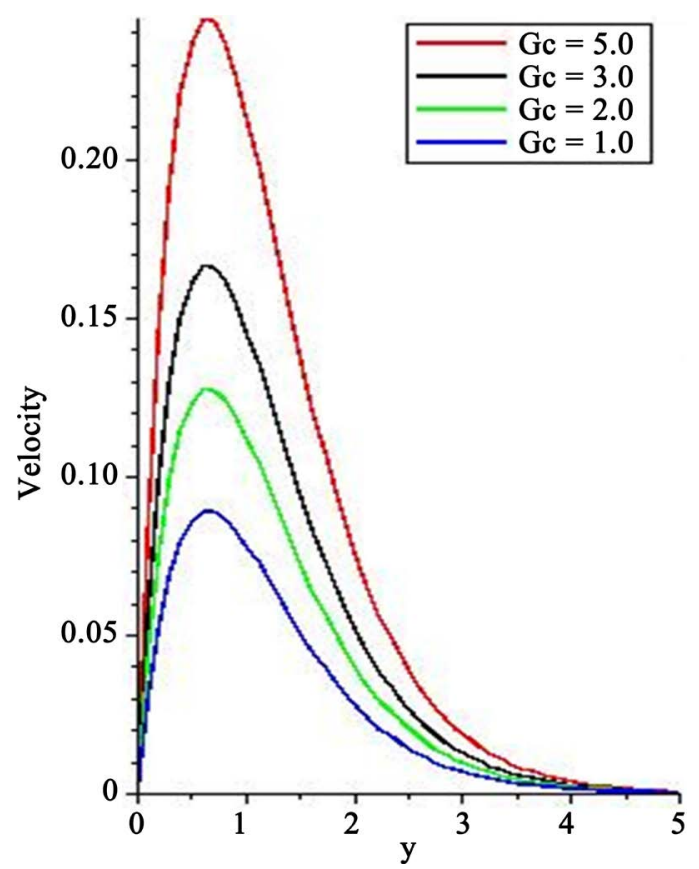

Figure 6. Velocity profiles for different values of $G c$.

Grashof number. Buoyancy force acts like a favourable pressure gradient which accelerates the fluid within the boundary layer.

\subsection{Temperature Profiles}

Figures 7-9 demonstrate the temperature profiles.

In Figure 7, the influence of Prandtl number on the temperature is shown. It is seen that, the temperature decreases when the Prandtl number is increased. It is justified due to the fact that thermal conductivity of the fluid decreases with 


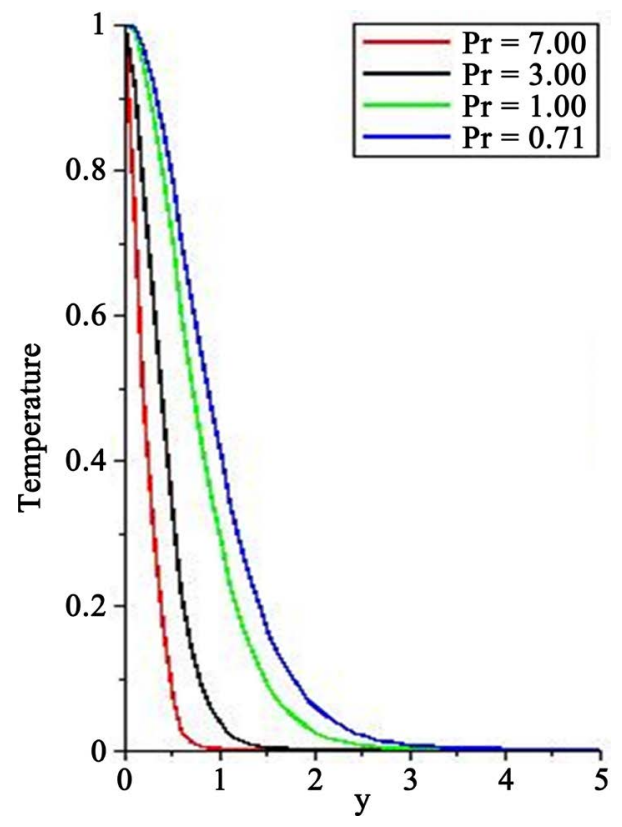

Figure 7. Temperature profiles for different values of Pr.

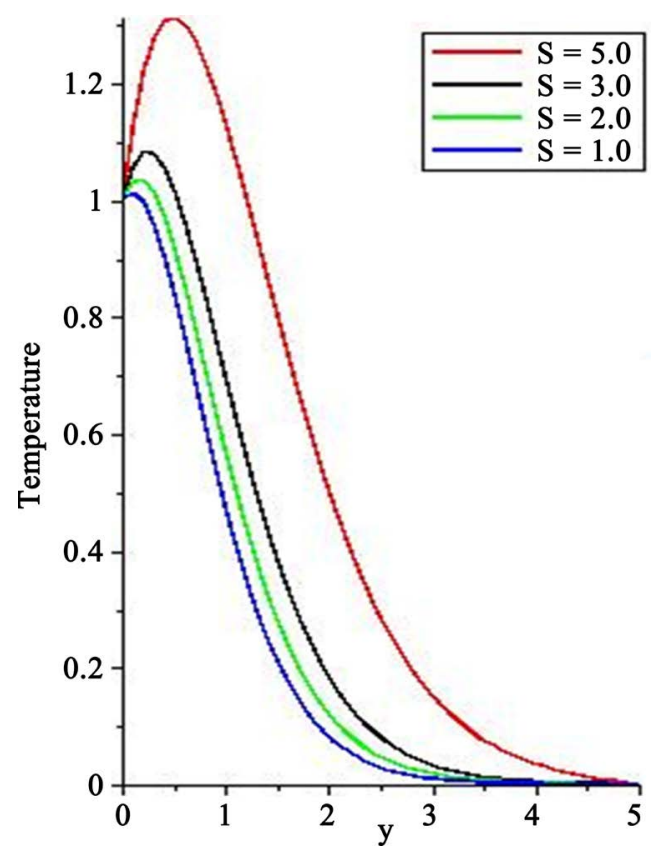

Figure 8. Temperature profiles for different values of $S$.

higher Prandtl number and hence retards the thermal boundary layer thickness. Figure 8 represents effect of heat sink on the temperature. It is depicted that, the temperature increases with increase in heat generation. Variation of suction parameter on the temperature is illustrated in Figure 9. It is observed that, the temperature decreases with increase in the suction parameter.

\subsection{Concentration Profiles}

Figures 10-12 display the concentration profiles. 


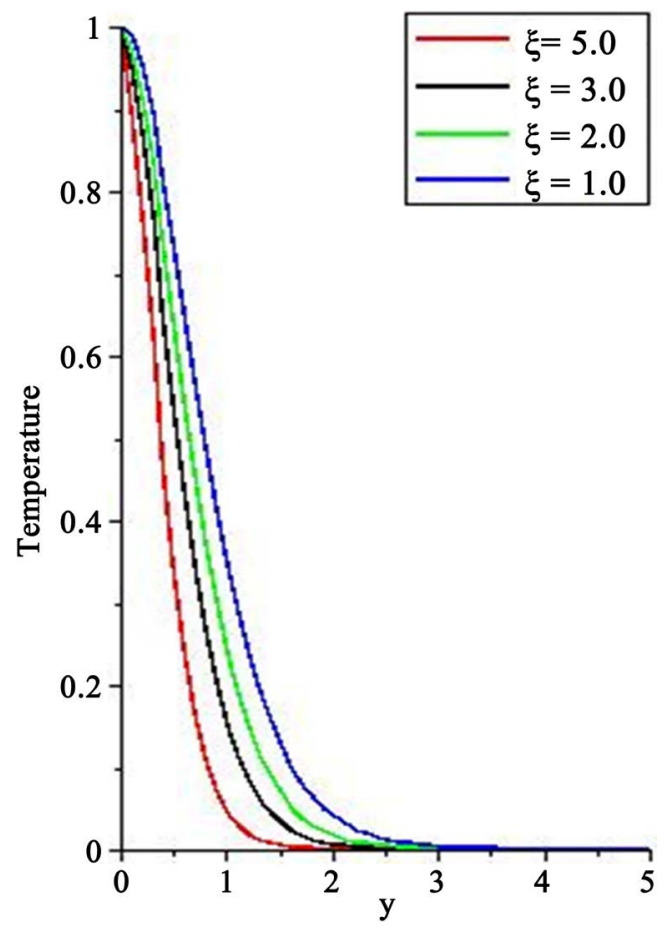

Figure 9. Temperature profiles for different values of $\xi$.

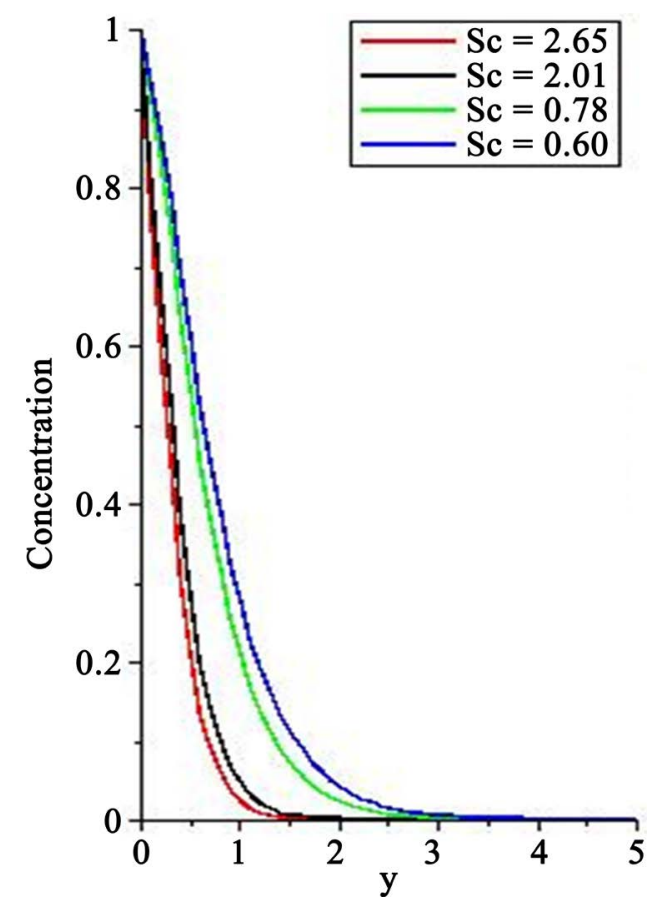

Figure 10. Concentration profiles for different values of $S c$.

Effect of Schmidt number on the concentration is presented in Figure 10. It is noted that, the concentration is lower due to increasing Schmidt number. This causes the concentration buoyancy effects to decrease yielding a reduction in the fluid concentration. The reductions in the velocity and concentration profiles are accompanied by simultaneous reductions in the velocity and concentration 
boundary layers. In Figure 11, the influence of chemical reaction parameter on the concentration is shown. It is demonstrated that. The concentration is lower as the chemical reaction parameter is increased. Figure 12 represents the variation of suction parameter on the concentration. It is seen that, the concentration deceases with increasing suction parameter.

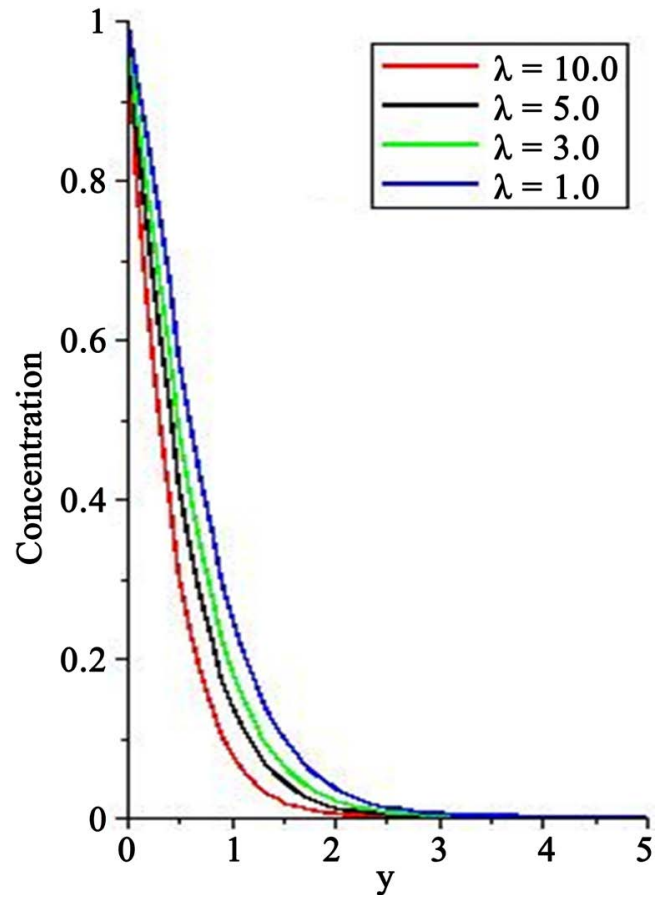

Figure 11. Concentration profiles for different values of $\lambda$.

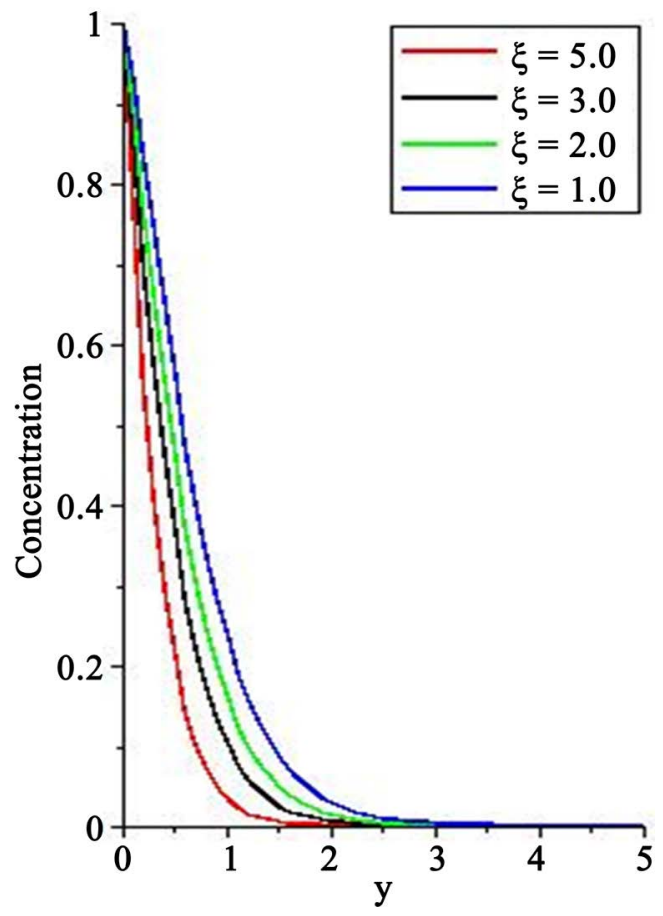

Figure 12. Concentration profiles for different values of $\xi$. 


\section{Conclusions}

Magnetohydrodynamic (MHD) convective boundary layer flow of a Casson fluid past an infinite vertical plate in the presence of heat generation and homogeneous was studied. The flow was governed by a modeled coupled nonlinear system of partial differential equations (PDEs) in dimensional form which is transformed into non-dimensional form using some non-dimensional variables. EFDM was used to approximate the velocity, temperature, concentration field. From the results obtained our findings are:

1) Momentum boundary layer thickness reduces when Casson parameter increases.

2) Increase in suction parameter leads to the decrease in the velocity, temperature and concentration of the fluid.

3) The velocity becomes higher as heat generation parameter is increased while chemical reaction parameter increase leads to the decrease in the concentration of the fluid.

4) Magnetic field retards the motion of the fluid within the boundary layer.

5) The velocity of the fluid rises with increasing values of thermal Grashof and mass Grashof numbers.

6) Momentum boundary layer thickness reduces when Casson parameter increases.

\section{Acknowledgements}

The authors are grateful to Tertiary Education Trust Fund (TETFUND), Abuja, Nigeria for funding this research work under the Institutional Based Research (TETFUND/DRSS/UNI/GUSAU/2015/RPVOL.1).

\section{Conflicts of Interest}

The authors declare no conflicts of interest regarding the publication of this paper.

\section{References}

[1] Kateria, H.R. and Patel, H.R. (2016) Radiation and Chemical Reaction Effects on MHD Casson Fluid Flow Past an Oscillating Vertical Plate Embedded in a Porous Medium. Alexandria Engineering Journal, 55, 583-595.

https://doi.org/10.1016/j.aej.2016.01.019

[2] Makinde, O.D. and Mhone, Y.P. (2005) Heat Transfer to MHD Oscillatory Flow in a Vertical Channel Filled with Porous Medium. Romanian Journal of Physics, 50, 931-938.

[3] Pushpalatha, K., Rahmana Reddy, J.V., Sugunamma, V. and Sandeep, N. (2017) Numerical Study of Chemically Reacting Unsteady Casson Fluid Flow Past a Stretching Surface with Cross Diffusion and Thermal Radiation. De Gruyter Open, 7, 69-76. https://doi.org/10.1515/eng-2017-0013

[4] Raju, C.S.K., Sandeep, N., Sugunamma, V., Babu, M.J. and Reddy, J.R. (2016) Heat and Mass Transfer in Magnetohydrodynamic Casson Fluid over an Exponentially 
Permeable Stretching Surface. Engineering Science and Technology, an International Journal, 19, 45-52. https://doi.org/10.1016/j.jestch.2015.05.010

[5] Kumaran, G., Sandeep, N. and Ali, M.E. (2017) Computational Analysis of Magnetohydrodynamic Casson and Maxwell Flows over a Stretching Sheet with Cross Diffusion. Results in Physics, 7, 147-155. https://doi.org/10.1016/j.rinp.2016.12.011

[6] Reddy, G.J., Raju, R.S. and Rao, J.A. (2017) Influence of Viscous Dissipation on Unsteady MHD Natural Convective Flow of Casson Fluid over an Oscillating Vertical Plate via FEM. Ain Shams Engineering Journal, 9, 1907-1915.

[7] Hayat, T., Shehzad, S. and Alsaedi, A. (2012) Soret and Dufour Effects on Magnetohydrodynamic (MHD) Flow of Casson Fluid. Applied Mathematics and Mechanics, 33, 1301-1312. https://doi.org/10.1007/s10483-012-1623-6

[8] Ajayi, T.M., Omowaye, A.J. and Animasaun, I.L. (2017) Viscous Dissipation Effects on the Motion of Casson Fluid over an Upper Horizontal Thermally Stratified Melting Surface of a Paraboloid of Revolution: Boundary Layer Analysis. Journal of Applied Mathematics, 2017, Article ID: 1697135. https://doi.org/10.1155/2017/1697135

[9] Raju, R.S., Reddy, B.M. and Reddy, G.J. (2017) Influence of Angle of Inclination on Unsteady MHD Casson Fluid Flow Past a Vertical Surface Filled by Porous Medium in Presence of Constant Heat Flux, Chemical Reaction and Viscous Dissipation. Journal of Nanofluids, 6, 668-679. https://doi.org/10.1166/jon.2017.1368

[10] Rajakumar, K.V.B., Balamurugan, K.S., Reddy, M.U. and Murthy, C.V. (2018) Radiation, Dissipation and Dufour Effects on MHD Free Convection Casson Fluid Flow through a Vertical Oscillatory Porous Plate with Ion-Slip Current. International Journal of Heat and Technology, 36, 494-508. https://doi.org/10.18280/ijht.360214

[11] Omokhuale, E. and Altine, M.M. (2017) Casson Fluid Flow and Mass Transfer along a Porous Medium Using Perturbation Method. The Journal of the Mathematical Association of Nigeria (Abacus), Mathematics Science Series, 44, 334-343.

[12] Raju, M.C., Reddy, N.A. and Varma, S.V.K. (2014) Analytical Solution of MHD Free Convective, Dissipative Boundary Layer Flow Past a Vertical Porous Surface in the Presence of Thermal Radiation, Chemical Reaction and Constant Suction. Ain Shams Engineering Journal, 5, 1361-1369. https://doi.org/10.1016/j.asej.2014.07.005

[13] Uwanta, I.J. and Omokhuale, E. (2014) Effects of Variable Thermal Conductivity on Heat and Mass Transfer with Jeffery Fluid. International Journal of Mathematical Archive, 5, 135-149. https://doi.org/10.1155/2014/291857

[14] Arthur, E.M., Seini, I.Y. and Bortteir, L.B. (2014) Analysis of Casson Fluid Flow over a Vertical Porous Surface with Chemical Reaction in the Presence of Magnetic Field. Journal of Applied Mathematics and Physics, 3, 713-723.

https://doi.org/10.4236/jamp.2015.36085

[15] Biswas, R., Afikuzzaman, M., Mondal, M. and Ahmed, S.F. (2018) MHD Convection and Heat Transfer Flow through a Vertical Plate in the Presence of Chemical Reaction. Frontiers in Heat and Mass Transfer, 11, 13. https://doi.org/10.5098/hmt.11.13

[16] Biswas, R., Mondal, M., Sarkar, D.R. and Ahmmed, S.F. (2017) Effects of Radiation and Chemical Reaction on MHD Unsteady Heat and Mass Transfer of Casson Fluid Flow Past a Vertical Plate. Journal of Advances in Mathematics and Computer Science, 23, 1-16. https://doi.org/10.9734/JAMCS/2017/34292

[17] Hellum, J.D. and Churchill, S.W. (1962) Transient and Steady State Free and Natural Convection, Numerical Solutions: Part 1. The Isothermal Vertical Plate. AIChE Journal, 8, 690-692. https://doi.org/10.1002/aic.690080525 
[18] Sharma, B.K., Yadav, K., Mishra, N.K. and Chaudhary, R.C. (2012) Soret and Dufour Effects on Unsteady MHD Mixed Convection Flow Past a Radiative Vertical Porous Plate Embedded in a Porous Medium with Chemical Reaction. Applied Mathematics, 3, 717-723. https://doi.org/10.4236/am.2012.37105

[19] Reddy, N.A. and Janardhan, K. (2017) Soret and Dufour Effects on MHD Casson Fluid over a Vertical Plate in Presence of Chemical Reaction and Radiation. International Journal of Current Research and Review, 9, 55-61.

https://doi.org/10.7324/IJCRR.2017.92411

[20] Ahmed, T. and Alam, M. (2013) Finite Difference Solution of MHD Mixed Convection Flow with Heat Generation and Chemical Reaction. Procedia Engineering, 56, 149-156. https://doi.org/10.1016/j.proeng.2013.03.101

[21] Mondal, M., Biswas, R., Shanchia, K., Hassan, M. and Ahmmed, S.F. (2019) Numerical Investigation with Stability Convergence Analysis of Chemically Hydromagnetic Casson Fluid Flow in the Effects of Thermophoresis and Brownian Motion. International Journal of Heat and Technology, 3, 59-70. https://doi.org/10.18280/ijht.370107

[22] Jasmine Benazir, A., Sivaraj, R. and Makinde, O.D. (2016) Unsteady Magnetohydrodynamic Casson Fluid Flow over a Vertical Cone and Flat Plate with Non-Uniform Heat Source/Sink. International Journal of Engineering Research in Africa, 21, 69-83. https://doi.org/10.4028/www.scientific.net/JERA.21.69

[23] Animasaun, I.L., Adebile, E.A. and Fagbade, A.I. (2016) Casson Fluid Flow with Variable Thermo-Physical Property along Exponentially Stretching Sheet with Suction and Exponentially Decaying Internal Heat Generation Using the Homotopy Analysis Method. Journal of the Nigerian Mathematical Society, 35, 1-17. https://doi.org/10.1016/j.jnnms.2015.02.001

[24] Mohan, S.R., Reddy, G.V. and Balakrishna, S. (2018) An Unsteady MHD Free Convection Flow of Casson Fluid Past an Exponentially Accelerated Infinite Vertical Plate through a Porous Media in the Presence of Thermal Radiation, Chemical Reaction and Heat Source or Sink. International Journal of Engineering and Techniques, 4, 16-27. 